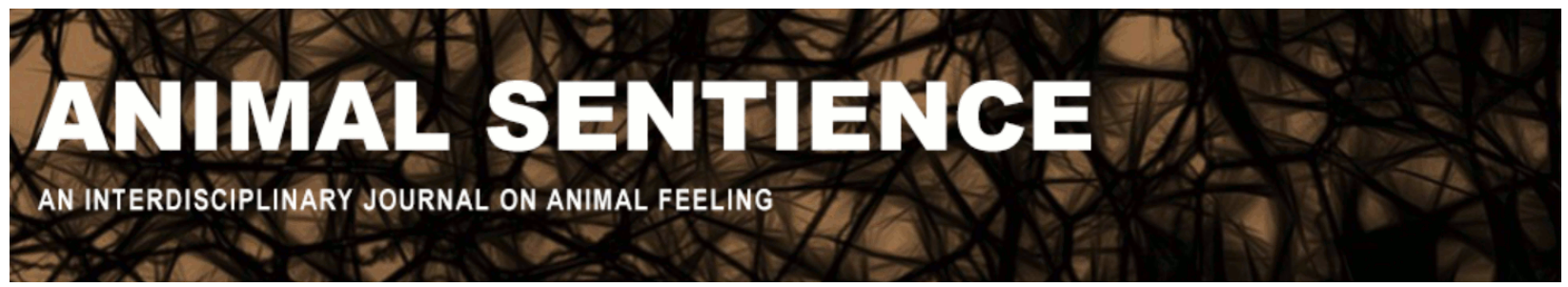

Sneddon, Lynne U.; Wolfenden, David C.C.; Leach, Matthew C.; Valentim, Ana M.; Steenbergen, Peter J.; Bardine, Nabila; Broom, Donald M.; and Brown, Culum (2018) Ample evidence for fish sentience and pain. Animal Sentience 21(17) DOI: $10.51291 / 2377-7478.1375$

Date of submission: 2018-10-12 Date of acceptance: 2018-11-27

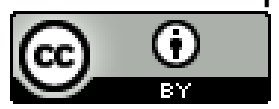




\section{Sneddon, Lynne U.; Wolfenden, David C.C.; Leach, Matthew C.; Valentim, Ana M.;}

Steenbergen, Peter J.; Bardine, Nabila; Broom, Donald M.; and Brown, Culum (2018) Ample evidence for fish sentience and pain. Animal Sentience 21(17)

DOI: $10.51291 / 2377-7478.1375$

Date of submission: 2018-10-12

Date of acceptance: $2018-11-27$

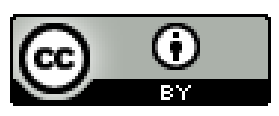

Authors

Lynne U. Sneddon, David C.C. Wolfenden, Matthew C. Leach, Ana M. Valentim, Peter J. Steenbergen, Nabila Bardine, Donald M. Broom, and Culum Brown 


\title{
Ample evidence for fish sentience and pain
}

Response to Commentary on Sneddon et al. on Sentience Denial

\section{Lynne U. Sneddon', David C.C. Wolfenden'2, Matthew C. Leach ${ }^{3}$, Ana M. Valentim ${ }^{4}$, Peter J. Steenbergen ${ }^{5}$, Nabila Bardine ${ }^{6}$, Donald M. Broom ${ }^{7}$ and Culum Brown ${ }^{8}$}

\author{
1Institute of Integrative Biology, University of Liverpool, UK \\ 2Blue Planet Aquarium, Cheshire, UK \\ ${ }^{3}$ School of Natural and Environmental Sciences, Newcastle University, UK \\ ${ }^{4}$ Institute of Molecular and Cell Biology, University of Porto, Portugal \\ ${ }^{5}$ Developmental Biology Unit, European Molecular Biology Laboratory, Germany \\ ${ }^{6}$ Holistic Life Coach, Heidelberg, Germany \\ ${ }^{7}$ Department of Veterinary Medicine, University of Cambridge, UK \\ ${ }^{8}$ Department of Biological Sciences, Macquarie University, Australia
}

\begin{abstract}
The majority of commentaries are supportive of our position on the scepticism that muddies the waters surrounding fish pain and sentience. There is substantial empirical evidence for pain in fish. Animals' experience of pain cannot be compared to artificial intelligence (AI) because AI can only mimic responses to nociceptive input on the basis of human observations and programming. Accepting that fish are sentient would not be detrimental to the industries reliant on fish. A more proactive discussion between scientists and stakeholders is needed to improve fish welfare for the benefit of all.
\end{abstract}




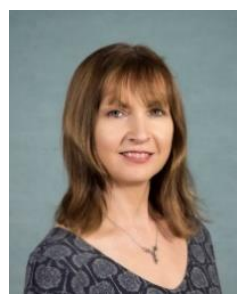

Lynne U. Sneddon, Director of Bioveterinary Science, University of Liverpool, was one of the first scientists to discover nociceptors that detect painful stimuli in fish. Sneddon has published empirical studies that drive the fish welfare agenda in many contexts. Website

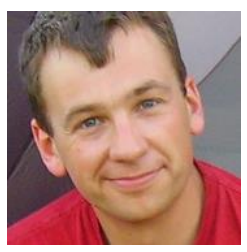

Matthew C. Leach is member of the Pain \& Animal Welfare Science Group at Newcastle University, which is internationally recognised for work on assessing and alleviating mammalian pain. Leach's current work gauges pain noninvasively through the use of grimace scales. Website

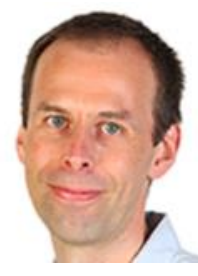

Peter I. Steenbergen has developed behavioural tests to assess stress and fear in in larval zebrafish. He described the ability of buprenorphine to alleviate the response of zebrafish larvae to chemical and electrical noxious stimuli. Website

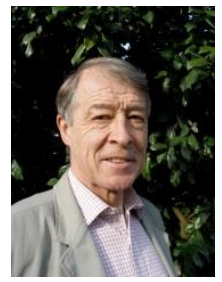

Donald M. Broom is Emeritus Professor of Animal Welfare in the Department of Veterinary Medicine, Cambridge University. His work has concerned sentience, sustainability and the welfare of a wide range of animals including fish. Website

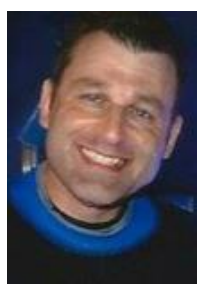

David C.C. Wolfenden, M.Phil in ornamental fish welfare, Curator at Blue Planet Aquarium, UK, is engaged in aquatic animal conservation and welfare research. Wolfenden is also a freelancer for ornamental-fish-keeping magazines and has authored chapters on fish welfare. Website

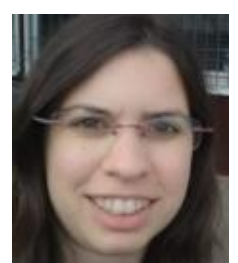

Ana M. Valentim, post-doctoral researcher at the Instituto de Investigação e Inovação em Saúde, Porto University, is specialised in Laboratory Animal Science. She studies anaesthesia side effects to refine anaesthesia in rodents and zebrafish models. Website

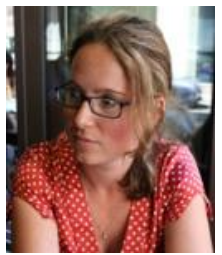

Nabila Bardine is a developmental biologist with a keen interest in animal behaviour and welfare. She is now a holistic therapist who helps people to lower their level of stress and gain wellbeing. Website

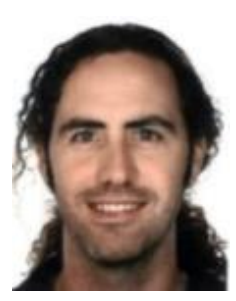

Culum Brown, Associate Professor of vertebrate evolution at Macquarie University, is Co-Editor of Fish Cognition and Behavior and Editor of the Journal of Fish Biology. He studies behavioural ecology of fishes with a special interest in cognition, personality and laterality. Website 
Most of the commentators are supportive of our position on scepticism about fish sentience and pain perception (Woodruff; Porcher; Jones; Meyers-Manor; Martin \& Gerlai; Manzotti; Demin et al.; da Silva et al.; Franks et al.). Woodruff points out that neurobiological evidence does exist for pain in fish. We would like to add that A delta fibres in fish are polymodal; they respond to the same stimuli as mammalian $\mathrm{C}$ fibres but conduct faster due to the myelin sheath (Sneddon 2015). In addition, brain gene expression studies in fish demonstrate that similar "pain" genes are involved (Herrero-Turrion et al. 2014; Reilly et al. 2008).

In agreement with Meyers-Manor, we note that even when the human central nervous system (CNS) is under development, the neurobiological system to respond to pain is there in babies; so even if fish have "only" analogous structures and larvae have an underdeveloped nervous system, pain and/or nociception mechanisms can also be there. Not so long ago, surgeons were denying pain relief to human babies because they could not feel pain (Segner 2016). We now know this is not true. To state the obvious: if an animal looks like it's in pain, assume it is until the contrary is empirically demonstrated.

Human pain perception did not appear spontaneously from nowhere. The pain system probably has ancient evolutionary roots given the important role it plays in preventing injury and death. However, pain is unlikely to be an identical experience across species since it is not even uniform across humans, who vary in threshold and experience. The question should not be "how similar is pain in fish and humans?" This would be anthropocentric - even though our own pain is all we have to go on. As with many other traits, evolutionary history, variation in selective forces and life history will shape pain perception for a particular species; hence comparing the quality of human and non-human animal pain may not be so useful. It is more meaningful to ask whether the pain state is important to a species in terms of deleterious consequences if damage is not detected or avoided. As Martin \& Gerlai point out, we use fish as models for pain in biomedical studies; the underlying assumption is that fish pain is relevant to human pain in its molecular biology, physiology and behavioural responses. Sceptics do not object to studies on stress, for example, where fish are a very important model species; yet, like pain, stress has a psychological component in humans. Science increasingly uses zebrafish as an alternative to laboratory rodents to understand human physiology and to test drugs for potential human applications (Demin et al.). The reason this is so widely accepted is the high degree of similarity between fish and human physiology. This underscores the need to give fish the appropriate ethical treatment when they are used in biomedical or other fields of research.

Adamo compares the responses to an animal experiencing pain to the responses that artificial intelligence (AI) programming could produce. There are a number of problems in this reasoning that Adamo does not consider. We agree with Porcher that computers just mimic what we know is a correct response to the situation we programme it to respond to. AI is programmed by us, and we design computers and robots to do things we would do; it is our subjective experience that grounds these responses. This has nothing to do with replicating the internal state of a creature with a complex nervous system that is the product of natural selection and evolution over eons. This is particularly evident when a stimulus triggers the expression of genes that may be further modified by epistatic or epigenetic mechanisms. Animals that do not show complex, cognitive responses to pain do not live for long. Without the negative affective component of pain, there would be no resulting long-term avoidance of dangerous stimuli/contexts. The computers that process all the information 
used for AI are powerful and considered very complex. Yet some consider animals like fish, who exhibit a similar range of complex actions without human programming, as not complex. Fish are able to exhibit stress, fear, anxiety, complex learning and selective actions, assigning priority to their needs as a function of the situation without being programmed by us. Adamo makes the important point that complex optogenetic and imaging techniques are needed to study the neural networks and pathways underlying the mechanisms of pain in fish.

Yokawa \& Baluška suggest that the effects of anaesthetics are similar in plants and fish. Like all living beings, plants have cells, so anaesthetics will dysregulate some mechanisms. But in fish, anaesthetics also act on brain receptors, which are absent in plants. When exposed to an anaesthetic, a Venus fly trap becomes unresponsive to touch stimulus (Yokawa et al. 2018); but to infer sentience in plants from this goes well beyond the data: Our target article does not conclude that fish are sentient just because they respond to anaesthetics. This is merely one of many converging lines of evidence that collectively support our conclusion (Walters). The evidence for sentience and pain in fish is by now abundant and clear (Broom 2014; Sneddon 2015; Brown 2017). To draw a parallel conclusion about plants more, complex experiments would be needed.

The conclusions from our evidence are fully supported by Franks et al. who commented that, rather than study whether fish experience pain (which has already been proven), there is now the need to study positive emotional experience in fish. Researchers have already started to do so in mammals with success (Mendl et al. 2008; LaFollette et al. 2017). This line of research would provide a fuller framework for appropriate fish welfare, not only preventing pain and poor welfare states but also providing positive experiences.

We agree with da Silva et al. that nociception is not enough for inferring pain; behavioural flexibility is necessary too. They use the term "nocifensive," which is a protective, instantaneous reflex response, usually an immediate withdrawal away from a noxious stimulus. This particular reflexive response does not show behavioural flexibility, but there are published examples of behavioural flexibility to pain in fish (review in Sneddon 2015).

"Consciousness" (better described as a level of awareness; Broom 2014) and sentience have been defined as being linked to a specific brain structure such as the multi-layered human cortex (Key 2016). Many others have argued that the cortex is not even the smoking gun of human consciousness (Manzotti; Devor 2016). A clinical study on human patients determined that those with disorders of consciousness had disrupted connections between the ventral anterior insula (AI) and the pregenual anterior cingulate cortex (pACC). The connections between brain areas seem to be more relevant than just the presence of particular brain areas (Fischer et al. 2016). There are different levels of awareness; examples of the more complex levels can be found in fish and cephalopods, whose brains are structurally different from those of mammals but have parallel neural mechanisms allowing similar functions. The kind of flexibility of function that these brains show with their sophisticated cognition and emotion is very different from that shown during machine learning. The lack of a particular brain structure is not valid grounds for denying animal awareness, pain or sentience.

Diggles \& Browman are sceptics about sentience and pain in fish, arguing that human brain structure is required for pain. In their commentary, they do not refute our specific claims that in their published critiques they misrepresent or misinterpret studies; they instead suggest that we are in the grip of "blind faith." We would reply that we are certainly 
not blind, but that we do have faith in robust, peer-reviewed, published empirical studies. We agree that scepticism is valuable, but only if the facts are correctly presented.

Whether the lives of fishes would change if it were broadly recognised that they are sentient and capable of suffering (Jacquet) is an important question. Fish are often described using a word best applied only to plants: as being "harvested." The term masks and minimizes the need to protect fish, by likening them to plants. Here we have a longstanding problem: fish are a commodity worth a lot of money. When there is money to be made, there will always be pressure to keep using fish the same way, with relatively little regard for their welfare. Jacquet also introduces the perspective of animal welfare in an environmental context and notes similarities with how CITES (Convention on International Trade in Endangered Species of Wild Fauna and Flora) classifies species: both endangered species and species that are accepted to experience pain may be left unprotected because of their economic value. Jacquet provides two excellent examples: The Southern Atlantic blue fin tuna and the Patagonian toothfish are both critically endangered and, based on the evidence, perceive pain. Yet, efforts to have them protected through CITES have failed, possibly due to their economic importance.

Many authors note that accepting that fish are sentient and feel pain may have socioeconomic, ethical, and, some suggest, negative consequences for the humans and industries that depend upon fish. That acknowledging fish pain would mean the end of the market for fish farmers in the developing world is exaggerated (Adamo). Similar arguments were made when animal welfare laws were brought in to protect terrestrial animals in industrial agriculture, but 50 years later, humans are still consuming around 60 billion birds and mammals per annum. The difference is that the doubt regarding fish sentience and pain makes the application of guidelines and refinement of techniques to further protect them very difficult. The kind of research aimed at improving welfare for fish as it did for terrestrial farm animals is still grossly underfunded. We suggest that this has to change. We are certainly not opposed to fish-reliant industries, but we argue that practices must be modified to take fish welfare into consideration as has been done with other species.

The process will involve small steps, requiring consultation with all stakeholders to see where we can improve both fish industries and fish welfare. Lessons from the legislative implementation for terrestrial animals will guide this process. Recent events in Australia, where the aquaculture industry worked alongside government welfare agencies to produce salmon products with an RSPCA tick of approval, demonstrate that this can be achieved. For example, it is well-known that stress reduces fish fillet quality (Bagni et al. 2007); thus, it is in the interests of fish food industries to consider ways to improve fish treatment.

As aquaculture production overtakes wild fish harvesting, there are further opportunities to improve fish welfare during rearing and slaughter. It is in the best interests of all those with a stake in using fish, including scientific research, that the welfare of these animals is safeguarded. By engaging fishers and the fish industries, we can communicate our empirical findings; one hopes that with discussion, consensus for moving forward can be reached. These are profitable industries, with jobs on the line, and fish production must meet society's demands. Contrary to Adamo's position that accepting fish sentience and applying the precautionary principle (Birch) would be detrimental, we propose that by working together, we can enhance welfare and productivity simultaneously. This would not only benefit humans in many ways, as has been demonstrated with terrestrial animal production (e.g., welfare assurance schemes; Buller et al. 2018), but it would benefit countless sentient creatures whose pain is currently being denied. 
Competing interests: The authors declare no competing interests.

Funding: The authors were not funded to write this Response.

\section{References}

Adamo, S. (2018) Sentience, the final frontier.... Animal Sentience 21(3).

Bagni, M., Civitareale, C., Priori, A., Ballerini, A., Finoia, M., Brambilla, G. and Marino, G. (2007) Pre-slaughter crowding stress and killing procedures affecting quality and welfare in sea bass (Dicentrarchus labrax) and sea bream (Sparus aurata). Aquaculture, 263(1), 52-60.

Birch, J. (2018) Degrees of sentience? Animal Sentience 21(11).

Broom, D.M. (2014) Sentience and Animal Welfare (pp. 200). Wallingford: CABI.

Brown, C. (2017) A risk assessment and phylogenetic approach. Animal Sentience 16(3).

Buller, H., Blokhuis, H., Jensen, P. and Keeling, L. (2018). Towards farm animal welfare and sustainability. Animals, 8(6), 81.

da Silva, M.L., Maximino, C. and Siqueira-Silva, D. H. (2018) Nocifensive behavior as evidence for sentient pain in fish. Animal Sentience 21(15).

Demin, K.A., Lakstygal, A.M. and Kalueff, A.V. (2018) Time to (finally) acknowledge that fish have emotionality and pain. Animal Sentience 21(13).

Devor, M. (2016) Where is pain in the brain? Animal Sentience 3(34).

Diggles, B. and Browman, H.I. (2018) Denialism and muddying the water or organized skepticism and clarity? THAT is the question. Animal Sentience 21(10).

Fischer, D.B., Boes, A.D., Demertzi, A. Evrard, H.C., Laureys, S., Edlow, B.L., Liu, H., Saper, C.B., Pascual-Leone, A., Fox, M.D. and Geerling, J.C. (2016) A human brain network derived from coma-causing brainstem lesions. Neurology, 87, 2427-2434.

Franks, B., Sebo, J. and Horowitz, A. (2018) Fish are smart and feel pain: What about joy? Animal Sentience 21(16).

Herrero-Turrión, M.J., Rodríguez-Martín, I., López-Bellido, R. and Rodríguez, R. (2014) Whole-genome expression profile in zebrafish embryos after chronic exposure to morphine: Identification of new genes associated with neuronal function and mu opioid receptor expression. BMC Genomics, 15, 874.

Jacquet, J. (2018) Defining denial and sentient seafood. Animal Sentience 21(8).

Jones, R.C. (2018) Fish sentience denial: Muddy moral water. Animal Sentience 21(5).

Key, B. (2016) Why fish do not feel pain. Animal Sentience 3(1).

LaFollette, M.R., O'Haire, M.E., Cloutier, S., Blankenberger, W.B. and Gaskill, B.N. (2017) Rat tickling: A systematic review of applications, outcomes, and moderators. PLoS ONE, 12(4), e0175320.

Manzotti, R. (2018) The human nervous system is not the gold standard for pain. Animal Sentience 21(12).

Martin, L. and Gerlai, R. (2018) Sentience: All or none or matter of degree? Animal Sentience 21(9).

Mendl, M., Burman, O.H., Parker, R.M. and Paul, E.S. (2009) Cognitive bias as an indicator of animal emotion and welfare: Emerging evidence and underlying mechanisms. Applied Animal Behaviour Science, 118(3-4), 161-181. 
Meyers-Manor, J.E. (2018) If it looks like a duck: Fish fit the criteria for pain perception. Animal Sentience 21(7).

Porcher, I.F. (2018) Fish sentience, consciousness, and AI. Animal Sentience 21(4).

Reilly, S.C., Quinn, J.P., Cossins, A.R. and Sneddon, L.U. (2008). Novel candidate genes identified in the brain during nociception in common carp (Cyprinus carpio) and rainbow trout (Oncorhynchus mykiss). Neuroscience Letters, 437, 135-138.

Segner, H. (2016) Why babies do not feel pain, or: How structure-derived functional interpretations can go wrong. Animal Sentience 3(26).

Sneddon, L.U. (2015) Pain in aquatic animals. Journal of Experimental Biology, 218, 967-976.

Sneddon, L.U., Lopez-Luna, J., Wolfenden, D.C.C., Leach, M.C., Valentim, A.M., Steenbergen, P.J., Bardine, N., Currie, A.D., Broom, D.M. and Brown, C. (2018) Fish sentience denial: Muddying the waters. Animal Sentience 21(1).

Walters, E.T. (2018) Defining pain and painful sentience in animals. Animal Sentience 21(14).

Woodruff, M.L. (2018) Pain in fish: Evidence from peripheral nociceptors to pallial processing. Animal Sentience 21(2).

Yokawa, K. and Baluška, F. (2018) Fish and plant sentience: Anesthetized plants and fishes cannot respond to stimuli. Animal Sentience 21(6).

Yokawa, K., Kagenishi, T., Pavlovič, A., Gall, S., Weiland, M., Mancuso, S. and Baluška, F. (2018) Anesthetics stop diverse plant organ movements, affect endocytic vesicle recycling, ROS homeostasis, and block action potentials in Venus flytraps. Annals of Botany, mcx155. 